\title{
AVALIAÇÃO DE ASFALTENOS PRECIPITADOS EM DIFERENTES CONDIÇÕES DE COMPOSIÇÃO, TEMPERATURA E PRESSÃO
}

\section{Lyzette Gonçalves Moraes de Moura*,\# e Paulo de Tarso Vieira e Rosa}

Instituto de Química, Universidade Estadual de Campinas, Cidade Universitária Zeferino Vaz, Barão Geraldo, 13083-970 Campinas $-\mathrm{SP}$, Brasil

Recebido em 19/07/2017; aceito em 02/10/2017; publicado na web em 04/12/2017

\begin{abstract}
EVALUATION OF ASPHALTENE PRECIPITATION IN DIFFERENT CONDITIONS OF COMPOSITION, TEMPERATURE, AND PRESSION. Asphaltenes are a heavy fraction of petroleum with the ability for self-association, a phenomenon that can occur spontaneously in the reservoirs or in any of the various stages of production and processing, depending on variations in pressure, temperature, composition and shearing, among others. In this work, it was evaluated the precipitation of asphaltenes in two oils ( $\mathrm{P} 01$ and $\mathrm{P} 02)$ in different conditions of temperature and pressure and in the absence or presence of $\mathrm{CO}_{2}$. Under high pressure and temperature conditions, the precipitation of asphaltenes was observed in the presence of $\mathrm{CO}_{2}$ in mixtures with methane, while the addition of propane induced precipitation in the absence of $\mathrm{CO}_{2}$. The results of analyses elemental (CHNO), SEM, FTIR and LDIMS were consistent with the structure assigned to samples of this nature (oils, maltenes and fractions of asphaltenes insoluble in n-pentane, C5I, and n-heptane, C7I).
\end{abstract}

Keywords: asphaltenes; precipitation; characterization; carbon dioxide; light alkanes.

\section{INTRODUÇÃO}

O petróleo é uma mistura complexa constituída de hidrocarbonetos saturados, aromáticos, resinas e asfaltenos. ${ }^{1,2} \mathrm{O}$ sistema polidisperso formado pelo petróleo normalmente está em condições de equilíbrio, mas perturbações como variações de composição, temperatura e pressão, podem induzir a precipitação dos asfaltenos..$^{2,3}$ Outros fatores como cisalhamento e efeitos eletrocinéticos aparentemente também exercem este efeito. . $^{1,4} 5$

Estudos indicam que a precipitação de asfaltenos também pode ocorrer espontaneamente nos reservatórios, levando à formação de nanopartículas, principalmente quando essa fração está presente em baixas concentrações $\left(<0,1 \mathrm{~g} \mathrm{~L}^{-1}\right) .^{6-8}$ Esse fenômeno, no entanto, normalmente está relacionado à produção/recuperação do petróleo, principalmente devido a variações de temperatura e à despressurização, comum nesses processos..$^{9-12} \mathrm{Na}$ prática, uma das razões mais frequentes para a ocorrência desse fenômeno in situ é a injeção de gases $\left(\mathrm{CO}_{2}, \mathrm{~N}_{2}\right.$, hidrocarbonetos leves, gás natural) nos processos de recuperação avançada, ou métodos especiais de recuperação do petróleo. ${ }^{10,13-16}$

Nos petróleos brasileiros são pouco comuns problemas de precipitação de asfaltenos, mas estudos indicam que este fenômeno deve ocorrer nos campos do pré-sal, devido à injeção de $\mathrm{CO}_{2}$ prevista para esses poços. ${ }^{12,17,16}$ Além de ser um fluido de injeção nos processos de recuperação, $\mathrm{o} \mathrm{CO}_{2}$ é um dos constituintes do gás natural e do próprio petróleo, geralmente presente em concentrações entre traços e 5\% (em mol),${ }^{18}$ podendo, contudo, chegar a concentrações mais elevadas, como cerca de $20 \%$ (em volume) nos reservatórios do pré-sal. ${ }^{19}$

Em alguns processos de recuperação do petróleo, a precipitação dos asfaltenos é desejada, como no VAPEX, um dos processos baseados no uso de solventes no qual propano (puro ou como componente majoritário de uma mistura de hidrocarbonetos leves)

*e-mail: lgmdemoura@gmail.com

\#Endereço atual: Departamento de Química, Universidade Federal do Maranhão, Campus do Bacanga, Avenida dos Portugueses, 1966, 65080-805 São Luís - MA, Brasil é injetado em reservatórios para a diluição e extração de petróleos pesados. ${ }^{14,20,21,22}$ A precipitação dos asfaltenos, contudo, normalmente é indesejável, pois leva a alterações na molhabilidade e permeabilidade originais da rocha reservatório, formação e estabilização de espumas e emulsões e obstrução de poços e tubulações das linhas de produção/processamento, por exemplo. ${ }^{8,11,14,15,21,23}$ Tais problemas têm levado ao desenvolvimento de aditivos, compostos cuja função é minimizar ou evitar a precipitação dos asfaltenos, sobretudo sem que seja necessária a interrupção da produção. ${ }^{24-26}$ Para tanto, é de fundamental importância conhecer as propriedades dessa fração e seu comportamento no petróleo.

Desta forma, este estudo tem como principal objetivo caracterizar asfaltenos precipitados com diferentes alcanos, na presença ou ausência de dióxido de carbono $\left(\mathrm{CO}_{2}\right)$, e variadas condições de temperatura e pressão.

\section{PARTE EXPERIMENTAL}

\section{Materiais}

Foram utilizados dois petróleos brasileiros de origens distintas, designados como P01 e P02; os gases dióxido de carbono $\left(\mathrm{CO}_{2}\right)$, metano $\left(\mathrm{CH}_{4}\right)$ e propano $\left(\mathrm{C}_{3} \mathrm{H}_{8}\right)$ (White Martins); e os hidrocarbonetos tolueno $\left(\mathrm{C}_{7} \mathrm{H}_{8}\right)$, n-heptano $\left(\mathrm{C}_{7} \mathrm{H}_{16}\right)$ e n-pentano $\left(\mathrm{C}_{5} \mathrm{H}_{12}\right)$ (Tedia Brazil), todos com pureza superior a $99,0 \%$.

\section{Métodos}

A análise elementar CHNO nos petróleos e frações (determinação dos teores de carbono, hidrogênio, nitrogênio e oxigênio) foi realizada no Instituto Federal do Espírito Santo (IFES, Brasil). Todos os demais procedimentos e análises foram realizados no Instituto de Química da Universidade Estadual de Campinas (IQ/UNICAMP, Brasil).

Extração de asfaltenos dos petróleos com n-pentano e n-heptano

A extração foi executada seguindo a norma IP143. ${ }^{27}$ No caso dos asfaltenos insolúveis em n-pentano, o procedimento foi o mesmo, 
mas este n-alcano foi utilizado em substituição ao n-heptano na etapa de precipitação.

Início de precipitação dos asfaltenos insolúveis em n-heptano nos petróleos pela técnica de espalhamento de laser

O sistema empregado na identificação do ponto de início de precipitação dos asfaltenos nos petróleos induzido pela adição de n-heptano em condições ambiente $\left(1\right.$ bar e $\left.28^{\circ} \mathrm{C}\right)$ foi aplicado conforme a metodologia desenvolvida e descrita por Marcano et al..$^{25}$

\section{Análise elementar CHNO nos petróleos e frações}

Foi utilizado um analisador elementar CHNS-932/VTF-900, Leco. A análise de cada amostra foi realizada em triplicata e o valor final é o resultado da média aritmética desses valores.

\section{Espectroscopia na região do infravermelho com transformada de Fourier (FTIR)}

Os espectros de FTIR foram obtidos em um espectrômetro de absorção na região do infravermelho com transformada de Fourier MB102, Bomem, no modo de transmitância, nas regiões de 600 - 4000 $\mathrm{cm}^{-1}$, com as amostras em solução em tolueno, analisadas na forma de um filme entre pastilhas de $\mathrm{NaCl}$.

Massa molar média dos petróleos e frações por espectrometria de massas com ionização por dessorção a laser (LDI-MS)

Foi utilizado um espectrômetro de massas Maldi Synapt HDMS, Waters, tipo QTOF, com laser ultravioleta e ajustado nas seguintes condições: laser com energia de 400 a.u., tensão de $20 \mathrm{~V}$ aplicada à placa, tensão de $10 \mathrm{~V}$ aplicada ao cone extrator, tensão de $10 \mathrm{~V}$ aplicada ao quadrupolo, $\mathrm{N}_{2}$ como gás de resfriamento com vazão de $10 \mathrm{~mL} \mathrm{~h}^{-1}$ e Ar como gás de colisão.

Para as análises, foram preparadas soluções estoque na concentração de $1 \mathrm{mg} \mathrm{mL}^{-1}$ de cada amostra em tolueno. Em seguida, $2 \mu \mathrm{L}$ de cada uma das soluções foram transferidos para a placa de análise, que foi deixada à temperatura ambiente e ao abrigo da luz, para volatilização do solvente, antes da execução das análises.

\section{Microscopia eletrônica de varredura (MEV) dos asfaltenos}

As análises foram realizadas em um microscópio eletrônico de varredura JSM-6340F, Jeol.

\section{Asfaltenos precipitados em sistemas contendo petróleo, $n$-alcanos leves $(n C<4)$ elou dióxido de carbono}

Neste trabalho foi utilizado um sistema de equilíbrio a alta pressão TharTech SPM20, Waters, composto por uma bomba hidráulica manual, um controlador de temperatura e uma célula de alta pressão; o sistema de filtração continha um elemento filtrante Swagelok de diâmetro médio de poros de 0,5 $\mu \mathrm{m}$. O esquema genérico é apresentado na Figura 1.

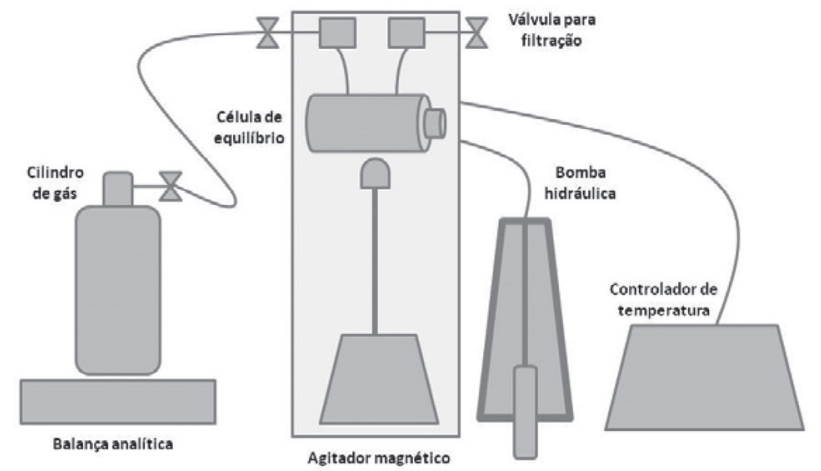

Figura 1. Diagrama esquemático do sistema de equilíbrio a alta pressão utilizado nos experimentos com petróleos, alcanos leves e dióxido de carbono
Uma barra magnética e uma quantidade conhecida de petróleo foram colocadas na célula de alta pressão, que foi conectada por meio de um tubo de PEEK a um cilindro de paintball contendo o gás desejado colocado sobre uma balança (Shimadzu, BL-3200H). Após a adição do gás, ou gases, o sistema de agitação foi ativado e a temperatura e pressão do equipamento foram ajustadas; estabelecido o equilíbrio temperatura/pressão, foi aguardado o tempo estipulado até à despressurização e, após o equilíbrio nas novas condições (mesma temperatura inicial e pressão inferior), foi aguardado $24 \mathrm{~h}$ e, então, foi realizada a filtração.

\section{RESULTADOS E DISCUSSÃO}

\section{Asfaltenos C5I e C7I dos petróleos P01 e P02 e Início de Precipitação}

A partir do procedimento realizado pela norma IP143, ${ }^{27}$ foram obtidos teores de $1,74 \pm 0,06 \%$ e $4,33 \pm 0,01 \%$ de asfaltenos insolúveis em n-pentano (C5I) e $0,44 \pm 0,00 \%$ e $2,45 \pm 0,07 \%$ de asfaltenos insolúveis em n-heptano (C7I) para os petróleos P01 e P02, respectivamente. Ambas as frações de asfaltenos estão presentes em maior percentual no petróleo P02, e os teores de asfaltenos C5I também são maiores nos dois petróleos. Pesquisas indicam que n-alcanos com cadeias menores, $\mathrm{nC}<7$, induziriam a precipitação de maior quantidade de asfaltenos de menor massa molar., ${ }^{3,21}$ Baixos teores de asfaltenos insolúveis em n-heptano indicam que esses petróleos apresentam maior concentração da fração de baixa massa molar (insolúvel em n-pentano, neste caso) e, segundo alguns autores, ${ }^{3,20,28}$ são mais susceptíveis a problemas de precipitação em campo. Dessa forma, o petróleo P02 estaria mais sujeito a tais problemas em relação ao P01.

$\mathrm{Na}$ determinação do início de precipitação de asfaltenos C7I nos petróleos (IP) por espalhamento de laser (1550 nm), as adições iniciais do n-heptano diluem a amostra e aumentam gradualmente a transmitância; a partir de certa concentração do n-alcano, há formação de agregados, que espalham a luz (laser); a partir deste ponto, enquanto ocorre a precipitação dos agregados formados, acréscimos subsequentes de n-heptano levam à formação de novos agregados e diluição da amostra, que reduzem o sinal de transmitância até um valor relativamente estável. O máximo do sinal da transmitância é o ponto de início da precipitação dos asfaltenos no petróleo, expresso como o volume de n-heptano por massa de petróleo $\left(\mathrm{mL} \mathrm{g}^{-1}\right){ }^{25}$

Neste trabalho, foram obtidos os valores de início de precipitação (IP, volume de n-heptano/massa de petróleo) de 2,65 \pm 0,07 $\mathrm{mL} \mathrm{g}^{-1}$ para o P01 e 1,30 $\pm 0,00 \mathrm{~mL} \mathrm{~g}^{-1}$ para o P02. Quanto menor o valor de IP, menor a quantidade de n-heptano necessária para induzir a precipitação, indicando que este petróleo está em uma condição mais sujeita a variações no equilíbrio do sistema, confirmando a interpretação dada aos teores das frações C5I e C7I desses petróleos.

\section{Composição elementar (CHNO) dos petróleos e frações C5 e C7 de asfaltenos e maltenos}

Os resultados da análise elementar (CHNO) dos asfaltenos C5I e C7I dos petróleos P01 e P02 e respectivos maltenos (C5S e C7S) são apresentados na Tabela 1 , junto aos valores da razão atômica H/C, sendo que maltenos é a denominação dada à fração do petróleo após a extração dos asfaltenos, isto é, à fração do petróleo solúvel no n-alcano utilizado na extração.

Nos estudos de petróleos e frações, geralmente os percentuais de carbono e hidrogênio são avaliados na forma da razão H/C: quanto menor for seu valor, maiores serão a massa molar e aromaticidade dos asfaltenos. ${ }^{3,29-32}$ Esta razão também pode estar relacionada ao início de precipitação dos asfaltenos e, neste caso, o maior valor de 
Tabela 1. Composição elementar (CHNO) dos petróleos P01 e P02 e respectivos asfaltenos (C5I e C7I) e maltenos (C5S e C7S) (percentual em massa) e razão atômica hidrogênio/carbono (H/C)

\begin{tabular}{|c|c|c|c|c|c|c|}
\hline \multirow{2}{*}{ Petróleo } & \multirow{2}{*}{ Fração } & \multicolumn{4}{|c|}{ Composição Elementar $(\%$ m/m) } & \multirow{2}{*}{$\begin{array}{c}\text { Razão Atômica } \\
\text { H/C }\end{array}$} \\
\hline & & $\mathbf{C}$ & $\mathbf{H}$ & $\mathbf{N}$ & $\mathbf{O}$ & \\
\hline \multirow{5}{*}{ P01 } & - & $86,80 \pm 0,70$ & $12,90 \pm 0,60$ & $0,43 \pm 0,01$ & $0,83 \pm 0,05$ & 1,77 \\
\hline & C5I & $85,00 \pm 2,00$ & $9,20 \pm 0,40$ & $1,83 \pm 0,08$ & $2,40 \pm 0,20$ & 1,29 \\
\hline & C7I & $86,00 \pm 0,60$ & $8,70 \pm 0,30$ & $1,96 \pm 0,05$ & $3,20 \pm 0,40$ & 1,21 \\
\hline & C5S & $88,00 \pm 4,00$ & $12,90 \pm 0,06$ & $0,26 \pm 0,03$ & $0,80 \pm 0,10$ & 1,75 \\
\hline & C7S & $86,50 \pm 0,30$ & $12,50 \pm 0,50$ & $0,26 \pm 0,03$ & $0,55 \pm 0,02$ & 1,72 \\
\hline \multirow{5}{*}{ P02 } & - & $84,60 \pm 0,90$ & $12,60 \pm 0,30$ & $0,50 \pm 0,10$ & $0,71 \pm 0,01$ & 1,77 \\
\hline & C5I & $86,00 \pm 0,50$ & $8,20 \pm 0,20$ & $2,00 \pm 0,01$ & $2,70 \pm 0,30$ & 1,14 \\
\hline & C7I & $86,00 \pm 1,00$ & $7,90 \pm 0,20$ & $2,13 \pm 0,02$ & $2,10 \pm 0,10$ & 1,09 \\
\hline & C5S & $87,50 \pm 0,50$ & $11,90 \pm 0,30$ & $0,15 \pm 0,02$ & $0,22 \pm 0,03$ & 1,62 \\
\hline & C7S & $87,40 \pm 0,20$ & $12,50 \pm 0,10$ & $0,25 \pm 0,01$ & $0,79 \pm 0,02$ & 1,70 \\
\hline
\end{tabular}

IP do petróleo P01, em relação ao P02, corrobora que este último seria mais susceptível à precipitação; além disso, os asfaltenos C7I do P02 apresentam menor razão H/C, concordando que seriam mais aromáticos e propensos à precipitação. ${ }^{33-35}$

Em relação aos heteroátomos $(\mathrm{N}, \mathrm{O}$ e $\mathrm{S})$, pesquisas indicam que estão concentrados nos asfaltenos e desempenham papel importante na autoassociação, pois normalmente fazem parte de suas porções polares, sítios em que ocorreriam ligações de hidrogênio e transferência de carga, interações envolvidas no processo de precipitação. ${ }^{2,36}$

Neste trabalho foram avaliados os percentuais de oxigênio $(\mathrm{O})$ e nitrogênio $(\mathrm{N})$ : ambos foram sempre maiores nos asfaltenos em relação aos maltenos correspondentes, concordando com resultados encontrados na literatura. ${ }^{2,33,34,36,37}$

\section{Precipitação de asfaltenos nos petróleos pela adição de n-alcanos leves $(\mathrm{nC}<4)$ e/ou dióxido de carbono}

Após os ensaios de caracterização inicial dos asfaltenos C5I e C7I dos petróleos $\mathrm{P} 01$ e P02, foram realizados experimentos em condições variadas de composição, temperatura e pressão. Foram adicionados metano e dióxido de carbono ao P01 (sistema $\mathrm{P} 01+\mathrm{CH}_{4}+\mathrm{CO}_{2}$, denominado S1) e somente propano ao P02 (sistema $\mathrm{P} 02+\mathrm{C}_{3} \mathrm{H}_{8}$, denominado S2).

A composição do primeiro sistema (S1) foi definida com o intuito de mimetizar o óleo vivo, que possui gás natural associado; o metano é o componente majoritário do gás natural, enquanto o dióxido de carbono, além de estar presente no mesmo, também é usado como fluido de injeção em processos de recuperação, nos quais podem ocorrer problemas de precipitação de asfaltenos. As condições de temperatura (T) e pressão (p) adotadas neste estudo para o sistema $\mathrm{S} 1$ foram estabelecidas por Cardoso et al. ${ }^{38}$ a partir de experimentos conduzidos com este petróleo (P01), nos quais foram definidas as curvas do ponto de bolha e de pressão de início de precipitação dos asfaltenos. Considerando-se que, para um determinado petróleo (ou sistema que o represente), a região entre essas curvas define as condições de temperatura e pressão nas quais há maior probabilidade de precipitação dos asfaltenos, ${ }^{15,16,21,38-40}$ os valores de T/p selecionados para este estudo foram tomados nesse intervalo.

Para o segundo sistema (S2), foi considerado um petróleo com maior teor de asfaltenos, que permitiria simular o processo de recuperação envolvendo a injeção de propano em reservatórios para a diluição e extração de petróleos pesados. Variações nas condições de T/p também foram utilizadas neste caso $\left(\mathrm{P} 02+\mathrm{C}_{3} \mathrm{H}_{8}\right)$, sendo que a composição foi selecionada com base no processo industrial de desasfaltação, que envolve condições críticas para o equilíbrio do sistema, como elevada razão propano/óleo. ${ }^{14,20-22} \mathrm{Na}$ Tabela 2 constam as condições nas quais foram conduzidos os respectivos experimentos.

Nos experimentos com ambos os sistemas, a precipitação ocorreu após a etapa de despressurização, tendo sido realizados testes de pressurização/despressurização/filtração em diversas condições. Convém observar que não foram realizados estudos quantitativos desses sistemas nesta etapa do trabalho, uma vez que se buscava confirmar as condições de precipitação e realizar a avaliação qualitativa dos asfaltenos assim obtidos.

No sistema S1, houve formação de agregados, provavelmente com diâmetro médio inferior a $0,5 \mu \mathrm{m}$, pois apenas uma pequena quantidade foi retida pelo elemento filtrante na etapa de filtração, executada após a despressurização; também não foi observada sedimentação de

Tabela 2. Condições dos experimentos com as misturas S1, sistema $\mathrm{P} 01+\mathrm{CH}_{4}+\mathrm{CO}_{2}$, e S2, sistema $\mathrm{P} 02+\mathrm{C}_{3} \mathrm{H}_{8}$ : temperatura $\left({ }^{\circ} \mathrm{C}\right)$; $\mathrm{p}_{\mathrm{i}}$, pressão inicial (bar); $\mathrm{p}_{\mathrm{f}}$, pressão após a despressurização (bar); e composição (percentual em massa, \% m/m)

\begin{tabular}{|c|c|c|c|c|c|c|c|c|c|c|c|c|c|}
\hline \multirow{2}{*}{$\underbrace{\text { Petróleo }}_{\text {Variável }}$ Condição } & \multicolumn{4}{|c|}{ P01 } & \multicolumn{9}{|c|}{ P02 } \\
\hline & 1 & 2 & 3 & 4 & 1 & 2 & 3 & 4 & 5 & 6 & 7 & 8 & 9 \\
\hline $\mathrm{T}$ & 47 & 47 & 58 & 80 & 47 & 50 & 50 & 50 & 60 & 60 & 120 & 120 & 120 \\
\hline$p_{i}$ & 550 & 550 & 550 & 310 & 550 & 400 & 400 & 400 & 140 & 70 & 450 & 450 & 400 \\
\hline $\mathrm{p}_{\mathrm{f}}$ & 400 & 400 & 380 & 210 & 400 & 70 & 70 & 70 & 33 & 15 & 240 & 70 & 70 \\
\hline Petróleo & 80,6 & 83,5 & 80,6 & 80,6 & 20 & 50 & 22,3 & 60 & 22,3 & 22,3 & 22,3 & 22,3 & 80 \\
\hline $\mathrm{CO}_{2}$ & 13,5 & 10,3 & 13,5 & 13,5 & - & - & - & - & - & - & - & - & - \\
\hline $\mathrm{CH}_{4}$ & 5,9 & 6,2 & 5,9 & 5,9 & - & - & - & - & - & - & - & - & - \\
\hline $\mathrm{C}_{3} \mathrm{H}_{8}$ & - & - & - & - & 80 & 50 & 77,7 & 40 & 77,7 & 77,7 & 77,7 & 77,7 & 20 \\
\hline
\end{tabular}


material no filtrado, indicando que a precipitação nesse sistema, nas condições avaliadas, é baixa, o que também pode estar relacionado ao baixo teor de asfaltenos determinado nessa amostra.

No S2, a precipitação ocorreu de forma bastante intensa, havendo, predominantemente, a sedimentação dos agregados no interior da célula de equilíbrio. Esse resultado era esperado, pois estudos indicam que a precipitação dos asfaltenos no interior dos reservatórios pode ocorrer nos processos de recuperação de petróleos pesados por injeção de solvente. ${ }^{14,21,22} \mathrm{Na}$ condição 9 , na qual foi utilizada a menor razão propano/óleo, também foi observada retenção de partículas no elemento filtrante, semelhante ao verificado com o S1; isto ocorreu provavelmente devido à maior quantidade de óleo no meio, que dispersou parte dos precipitados, evitando sua agregação e sedimentação.

Amostras obtidas de alguns desses experimentos foram submetidas a análises de MEV, FTIR e LDI-MS, juntamente às amostras dos asfaltenos C5I e C7I (norma IP143) dos respectivos petróleos. Os resultados foram comparados e são apresentados nas seções seguintes.

\section{Microscopia eletrônica de varredura (MEV) dos asfaltenos dos petróleos P01 e P02}

As imagens obtidas por microscopia eletrônica de varredura (MEV) dos asfaltenos C5I e C7I do P01 e P02 e de amostras S1 e S2 constam na Figura 2.

Os asfaltenos C5I e C7I dos petróleos $\mathrm{P} 01$ e $\mathrm{P} 02$ revelam estruturas regulares, relativamente bem definidas. $\mathrm{O}$ aspecto dos asfaltenos C7I condiz com os resultados da literatura, ${ }^{14,20,41}$ mas asfaltenos C5I apresentariam uma superfície menos regular e porosa, atribuída principalmente à remoção de resinas no processo de extração. Como o procedimento experimental de precipitação seguido foi análogo ao apresentado nos referidos trabalhos (IP143), é possível que haja dependência do teor de resinas e, nos petróleos avaliados, pode não ter havido o suficiente dessa fração para impedir a formação de estruturas regulares nos asfaltenos C7I e C5I. ${ }^{20,41}$

Por outro lado, os asfaltenos provenientes dos sistemas mais complexos se apresentaram como filmes, nos quais não foi possível observar, por MEV, estruturas definidas. Tal característica pode estar relacionada ao maior teor de asfaltenos de menor massa molar média presente nessas amostras $(<\mathrm{C} 5 \mathrm{I})$, que apresentam maior viscosidade em comparação àquelas extraídas pelo método IP143, ou, ainda, à presença de resíduos do próprio petróleo. ${ }^{28}$

\section{Espectroscopia na região do infravermelho com transformada de Fourier (FTIR)}

Os espectros obtidos por FTIR dos petróleos P01 e P02 e seus respectivos asfaltenos constam na Figura $3(\mathrm{a}, \mathrm{b})$.

Como pode ser observado na Figura 3 os espectros dos asfaltenos e dos respectivos petróleos apresentam, de modo geral, diversas semelhanças entre si, concordando com dados da literatura. ${ }^{42-45}$ No P01, a fração C5I apresenta bandas mais intensas de carbonos aromáticos em comparação à C7I, com uma banda correspondente a ligações com heteroátomos ( $\mathrm{N} \mathrm{e} \mathrm{O}$ ) na região de $3250 \mathrm{~cm}^{-1}$; contudo, ao comparar os precipitados obtidos em T/p elevados, essa banda é mais nítida no precipitado S2. Isso pode indicar que os heteroátomos estão predominantemente ligados aos anéis aromáticos no P01, enquanto no P02 essas ligações podem ser periféricas, com maior ocorrência nas cadeias laterais; no caso do precipitado do S2, como a precipitação é mais intensa, é possível que as subfrações mais estáveis em condições ambiente, precipitem durante a variação das condições de temperatura e pressão.

Bandas na região de $\mathrm{CH}$ alifáticos $\left(3000-2780 \mathrm{~cm}^{-1}\right)$ indicam predominância de hidrocarbonetos cíclicos alifáticos, comum em petróleos de base naftênica, como o P01.41,46

As amostras de asfaltenos apresentaram bandas relativas aos grupos aromáticos $(\mathrm{CH})$ na região de $1620-1590 \mathrm{~cm}^{-1}$ e aos grupos metil e metileno $(\mathrm{CH})$ na faixa de $1500-1350 \mathrm{~cm}^{-1}$; uma banda em $1462 \mathrm{~cm}^{-1}$ está provavelmente relacionada à deformação angular simétrica do grupo metileno $\left(\mathrm{CH}_{2}\right)$ em hidrocarbonetos; uma banda em $1456 \mathrm{~cm}^{-1}$ está próxima à banda de deformação angular assimétrica do grupo metila $\left(\mathrm{CH}_{3}\right)$; bandas em $1376 \mathrm{~cm}^{-1}$ sugerem vibração de deformação angular simétrica do grupo metila. ${ }^{41,47}$

Em torno de $1000 \mathrm{~cm}^{-1}$ são encontradas bandas relacionadas a funções oxigenadas, ${ }^{42,47}$ sendo que na faixa de $1030-1040 \mathrm{~cm}^{-1}$ estão bandas específicas de grupos sulfóxidos $(\mathrm{S}=\mathrm{O}){ }^{36,46,48}$

Bandas em torno de $720 \mathrm{~cm}^{-1}$, mais intensas nas amostras do P01,

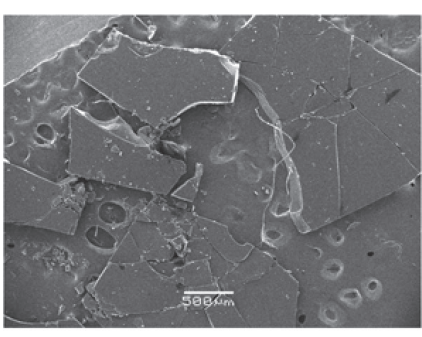

(a)

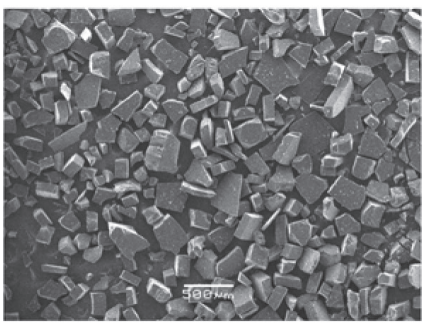

(d)

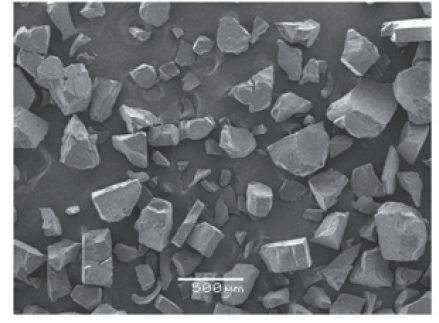

(b)

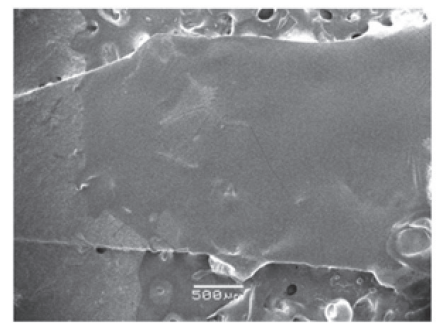

(e)

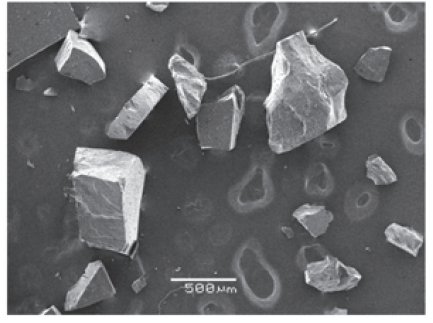

(c)

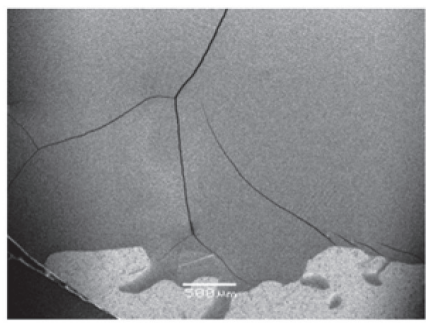

(f)

Figura 2. Imagens de microscopia eletrônica de varredura (MEV) dos asfaltenos (a) C5I/P01; (b); C5I/P02 (c); C7I/P01; (d) C7I/P02; (e) amostra do sistema S1na condição 1 (mistura $\mathrm{PO} 1+\mathrm{CH}_{4}+\mathrm{CO}_{2}, 80,6 \% \mathrm{~m} / \mathrm{m}$ de petróleo, à temperatura constante de $47^{\circ} \mathrm{C}$ e filtração após despressurização de 550 bar a 400 bar); (f) amostra do sistema $\mathrm{S} 2$ na condição 3 (mistura $\mathrm{P} 02+\mathrm{C}_{3} \mathrm{H}_{8}, 22,3 \% \mathrm{~m} / \mathrm{m}$ de petróleo, à temperatura constante de $50{ }^{\circ} \mathrm{C}$ e filtração após despressurização de 400 bar a 70 bar) (condições experimentais apresentadas na Tabela 2 ) 


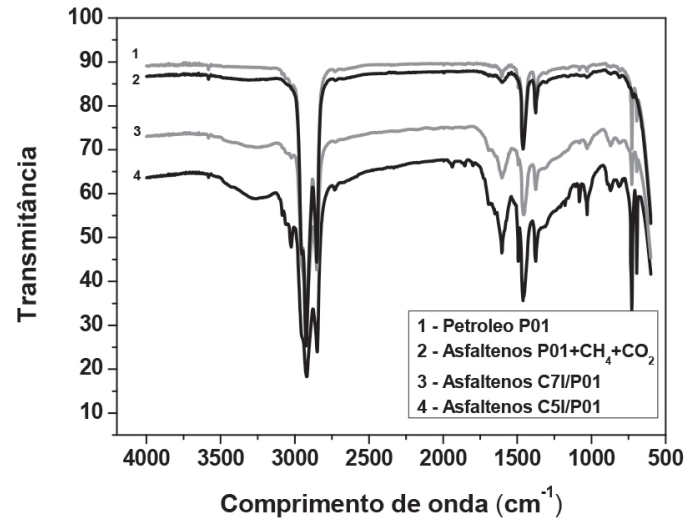

(a)

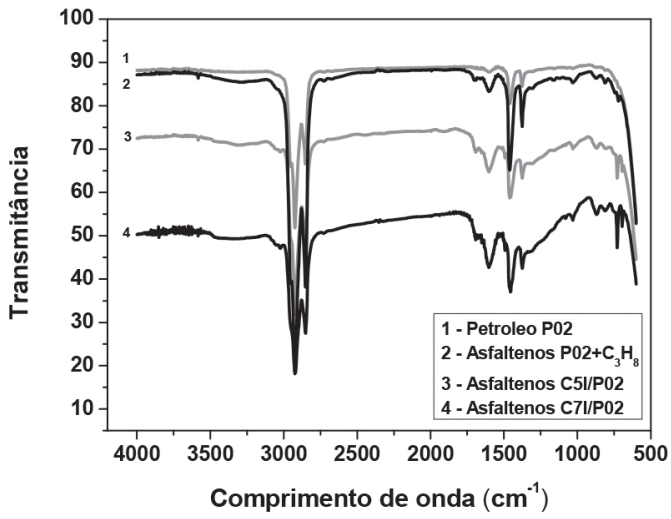

(b)

Figura 3. Espectros de absorbância na região do infravermelho: (a) petróleo P01 e asfaltenos C5I e C7I (norma IP143) e precipitado de um experimento com o sistema $\mathrm{PO1}+\mathrm{CH}_{4}+\mathrm{CO}_{2}$; e (b) petróleo $\mathrm{PO2}$ e asfaltenos $\mathrm{C5I}$ e C7I (norma IP143) e precipitado de um experimento com o sistema P02 $+\mathrm{C}_{3} \mathrm{H}_{8}$

correspondem, à presença do grupo metileno em cadeias alifáticas longas; já a banda em $694 \mathrm{~cm}^{-1}$, presente nos asfaltenos obtidos pela norma IP143, pode estar relacionada a um anel aromático monossubstituído, como o fenol. ${ }^{48}$

\section{Massas molares médias de petróleos e frações por} espectrometria de massas com ionização por dessorção a laser (LDI-MS)

As massas molares médias dos petróleos, frações de asfaltenos C5I e C7I e maltenos C5S e C7S foram obtidas por LDI-MS. Todos os espectros apresentaram o mesmo perfil e o espectro de massas dos asfaltenos C7I do petróleo P01 é apresentado na Figura 4. Pode ser observado que essa amostra possui uma distribuição da razão massa/ carga (m/z) típica de um sistema polidisperso, com um perfil monomodal e contínuo, isto é, sem intervalos de $\mathrm{m} / \mathrm{z}$ iguais ou próximos a zero. Essa característica é condizente com espectros encontrados em outros trabalhos para amostras de diferentes origens e obtidos por outros métodos da espectrometria de massas. ${ }^{42,49-54}$

As massas molares médias dos petróleos obtidas por LDI-MS foram 622 Da e 643 Da para os petróleos P01 e P02, respectivamente. As massas molares médias das frações resultantes das extrações com n-pentano (C5I/ C5S) foram sempre inferiores àquelas com n-heptano (C7I/ C7S): 592/ 579 Da para as frações C5 do P01 e 745/ 621 Da para as do P02 contra 759/ 615 Da para as frações C7 do P01 e 814/ 651 Da para as do P02. Tal comportamento era esperado, uma vez que, em relação aos asfaltenos, a fração de maltenos concentra as subfrações do petróleo de massas molares médias menores.

As massas molares médias das amostras de asfaltenos obtidas dos sistemas S1 e S2 nas diferentes condições trabalhadas (Tabela 2) resultaram em valores próximos entre si, sendo as médias aritméticas aproximadamente iguais a $396 \mathrm{Da}$ e $438 \mathrm{Da}$, respectivamente; esses valores são inferiores aos dos petróleos e aos de suas frações C5I e C7I, conforme esperado em função das menores cadeias carbônicas dos alcanos utilizados. ${ }^{3,21}$

De modo geral, os valores médios de massa molar para asfaltenos, principalmente C7I, encontrados neste estudo são próximos à massa molar atualmente considerada como a mais provável para essa fração, em torno de $750 \mathrm{Da}^{37,42,52-54}$

\section{CONCLUSÃO}

Os petróleos P01 e P02 apresentaram teores de asfaltenos relativamente baixos, sendo os percentuais de asfaltenos C7I inferiores

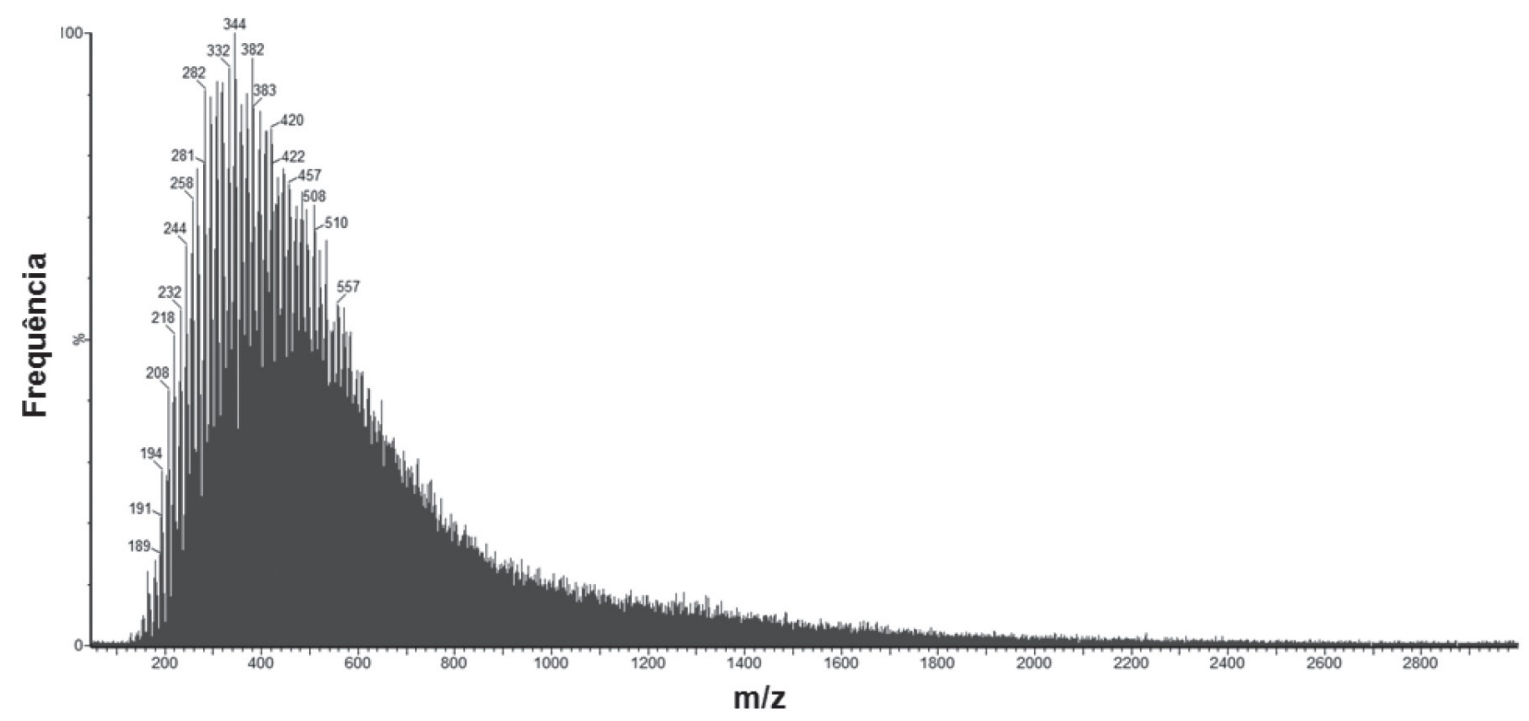

Figura 4. Espectro de massas dos asfaltenos C7I (insolúveis em n-heptano) do petróleo P01 obtidos por espectrometria de massas com ionização por dessorção a laser (LDI-MS) 
aos da fração C5I. O IP dos petróleos determinado por espalhamento de laser indicou que o P02, com menor IP, seria mais susceptível a problemas de precipitação de asfaltenos.

A análise elementar (CHNO) confirmou que há pouca variação nos teores de $\mathrm{C}$ e $\mathrm{H}$ dos petróleos, havendo maior variação em relação aos asfaltenos C5I e C7I, que são mais aromáticos que os C5I de acordo com a razão H/C. Os valores de massa molar obtidos por LDI-MS ficaram em torno de $600 \mathrm{Da}$, para os petróleos, e na faixa de 500 - 800 Da para os principais asfaltenos avaliados (C5I e C7I).

Os experimentos em condições de pressão e temperatura elevadas ( $\mathrm{p} \geq 50$ bar e $\mathrm{T} \geq 45^{\circ} \mathrm{C}$ ) com os sistemas $\mathrm{P} 01+\mathrm{CO}_{2}+\mathrm{CH}_{4}$ e $\mathrm{P} 02+\mathrm{C}_{3} \mathrm{H}_{8}$ mostraram que a precipitação proporcionada pela despressurização pode levar à formação de partículas com diâmetro inferior a $0,5 \mu \mathrm{m}$.

\section{AGRADECIMENTOS}

Os autores agradecemà CAPES (Coordenação de Aperfeiçoamento de Pessoal de Nível Superior), pela bolsa de doutorado concedida a L. G. M. de Moura, e à PETROBRAS (Petróleo Brasileiro S. A.), pelas amostras de petróleo fornecidas para o desenvolvimento deste trabalho.

\section{REFERÊNCIAS}

1. Behbahani, T. J.; Pet. Sci. 2016, 13, 155.

2. Pantoja, P. A.; Mendes, M. A.; Nascimento, C. A. O.; J. Pet. Sci. Eng. 2013, 109, 198.

3. Mansur, C. R. E.; Melo, A. R.; Lucas, E. F.; Energy Fuels 2012, 26, 4988.

4. Akbarzadeh. K.; Eskin, D.; Ratulowski, J.; Taylor, S.; Energy Fuels 2012, 26, 495.

5. Karan, K.; Hammami, A.; Flannery, M.; Stankiewicz, B. A.; Pet. Sci. Technol. 2003, 21, 629.

6. Betancourt, S. S.; Dubost, F. X.; Mullins, O. C.; Cribbs, M. E.; Creek, J. L.; Mathews, S. G.; International Petroleum Technology Conference, Dubai, UAE, 2007.

7. Dolomatov, S. A.; Shutkova, S. A.; Dezortsev, S. V.; J. Struct. Chem. 2012, 53, 563 .

8. Ghosh, A. K.; Chaudhuri, P.; Kumar, B.; Panja, S. S.; Fuel 2016, 185, 541.

9. Bahrami, P.; Kharrat, R.; Mahdavi, S.; Ahmadi, Y.; James, L.; Korean J. Chem. Eng. 2015, 32, 316.

10. Fard, S. R.; Khadar, R. H.; Energy Sources, Part A 2012, 34, 1868.'

11. Mckenna, A. M.; Marshall, A. G.; Rodgers, R. P.; Energy Fuels 2013, 27, 1257.

12. Mello, S. F.; Dissertação de Mestrado, Universidade Estadual de Campinas, Brasil, 2011.

13. Moradi, S.; Rashtchian, D.; Ghazvini, M. G.; Emadi, M. A.; Dabir, B.; Iran. J. Chem. Chem. Eng. 2012, 31, 89.

14. Luo, P.; Gu., Y; Fluid Phase Equilib. 2009, 277, 1.

15. Soroush, S.; Straver, E. J. M.; Rudolph, E. S. J.; Peters, C. J.; Loos, T. W.; Zitha, P. L. J.; Vafaie-Sefti, M.; Fuel 2014, 137, 405.

16. Oliveira, V. R. R.; Silva, N. A. E.; Souza, M. M. S.; Melo, S. A. B. V.; Costa; G. M. N.; Can. J. Chem. Eng. 2015, 93, 1697.

17. Hernández, J. A. B.; Tese de Doutorado, Universidade Estadual de Campinas, Brasil, 2013.

18. Fundamentos de Engenharia de petróleo; Thomas, J. E., org.; 1ª ed., Interciência: Rio de Janeiro, 2001.

19. Costa, I. V. L.; Dissertação de Mestrado. Universidade Federal do Rio de janeiro, Brasil, 2009.

20. Luo, P.; Wang, X.; Gu, Y.; Fluid Phase Equilib. 2010, 291, 103.

21. Arciniegas, L. M.; Babadagli, T.; Fuel 2014, 124, 202.

22. Pourabdollah, K.; Mokhtari, B. Fuel 2013, 107, 1.
23. Wang, W.; Taylor, C.; Hu, H.; Humphries, K. L.; Jaini, A.; Kitimet, M.; Scott, T.; Stewart, Z.; Ulep, K. J.; Houck, S.; Luxon, A.; Zhang, B.; Miller, B.; Parish, C. A.; Pomerantz, A. E.; Mullins, O. C.; Zare, R. N.; Energy Fuels 2017, 31, 9140.

24. Mena-Cervantes, V. Y.; Hernández-Altamirano, R.; BuenrostroGonzález, E.; Beltrán, H. I.; Zamudio-Rivera, L.; Energy Fuels 2011, 25, 224.

25. Marcano, F.; Moura, L. G. M.; Cardoso, F. M. R.; Rosa, P. T. V.; Energy Fuels 2015, 29, 2813.

26. Lowry, E.; Sedghi, M.; Goual, L.; Energy Fuel 2016, 30, 7187.

27. IP143/01. Standard methods for analysis and testing of petroleum and related products, 2001.

28. Deo, M.; Parra, M.; Energy Fuels 2012, 26, 2672.

29. Durand, E.; Clemancey, M.; Lancelin, J. M.; Verstraete, J.; Espinat, D.; Quoineaud, A. A.; Energy Fuels 2010, 24, 1051.

30. Nazar, A.R.S.; Bayandory, L.; IJChE 2008, 5 , 3.

31. Ovalles, C.; Rogel, E.; Moir, M.; Thomas, L.; Pradhan, A.; Energy Fuels 2012, 26, 549.

32. The chemistry and technology of petroleum; Speight, J. G., ed.; $4^{\text {th }}$ ed., Taylor \& Francis: Boca Raton, 2006.

33. Aguiar, J. I. S.; Garreto, M. S. E.; González, G.; Lucas, E. F.; Mansur, C. R. E.; Energy Fuels 2014, 28, 409.

34. Gawel, B.; Eftekhardadkhah, M.; Oye, G.; Energy Fuels 2014, $28,997$.

35. Leyva, C.; Ancheyta, J.; Berrueco, C.; Millán, M.; Fuel Process. Technol. 2013, 106, 734.

36. Daaou, M.; Bendedouch, D.; Modarressi, A.; Rogalski, M.; Energy Fuels 2012, 26, 5672.

37. Fossen, M.; Kallevik, H.; Knudsen, K. D.; Sjoblom, J.; Energy Fuels 2011, 25, 3552.

38. Cardoso, F.; Carrier, H.; Rosa, P.; Pauly, J.; Daridon, J. L.; 13th International Conference on Petroleum Phase Behaviour and Fouling, Saint-Petersburg, U. S. A., 2012.

39. Gonzalez, D. L.; Vargas, F. M.; Hirasaki, G. J.; Chapman, W. G.; Energy Fuels 2008, 22, 757.

40. Vargas, F. M.; Gonzalez, D. L.; Creek, J. L.; Wang, J.; Buckley, J.; Hirasaki, G. J.; Chapman, W. G.; Energy Fuels 2009, 23, 1147.

41. Davarpanah, L.; Vahabzadeh, F.; Dermanaki, A.; Oil Gas Sci. Technol. 2015, 70, 1035.

42. Poveda-Jaramillo, J.-C.; Molina-Velasco, D.-R.; Bohorques-Toledo, N.A.; Torres, M.-H.; Ariza-Léon, E.; CT\&F, Cienc., Tecnol. Futuro 2016, 6, 105.

43. Mohammadi, M.; Akbari, M.; Fakhroueian, Z.; Bahramian, A.; Azin, R.; Arya, S.; Energy Fuels 2011, 25, 3150.

44. Petrova, L. M.; Abbakumova, N. A.; Foss, T. R.; Romanov, G. V.; Pet. Chem. 2011, 51, 252.

45. Wang, X.; Gu, Y.; Energy Fuels 2011, 25, 5232.

46. Silverstein, R. M.; Webster, F. X.; Kimle, D. J.; Identificação espectrométrica de compostos orgânicos, $7^{\mathrm{a}}$ ed.; LTC: Rio de Janeiro, 2013.

47. Castro, L. V.; Vazquez, F.; Energy Fuels 2009, 23, 1603.

48. Adebiyi, F. M.; Thoss, V.; Fuel 2014, 118, 426.

49. Sabbah, H.; Morrow, A. L.; Pomerantz, A. E.; Zare, R. N.; Energy Fuels 2011, 25, 1597.

50. Wu, Q.; Seifert, D. J.; Pomerantz, A. E.; Mullins, O. C.; Zare, R. N.; Energy Fuels 2014, 28, 3010.

51. Liu, Y.-J.; Li, Z.-F.; J. Chem. 2015, 1.

52. Pomerantz, A. E.; Wu, Q.; Mullins, O. C.; Zare, R. N.; Energy Fuels 2015, 29, 2833.

53. Abdallah, W.; Pomerantz, A. E.; Sauerer, B.; Mullins, O. C.; Buiting, J.; JSPE Middle East Oil Gas Show and Conference, Manama, Kingdom of Bahrain, 2017.

54. Mullins, O. C.; Annu. Rev. Anal. Chem. 2011, 4, 393. 